\title{
The Algebraic Construction of Commutative Group
}

\author{
Yanyan Shan \\ Department of Mathematics, School of Science, Inner Mongolia University of Technology \\ Hohhot 010052, China \\ E-mail: bigeye_mm@163.com
}

\begin{abstract}
The construction of the integers introduced by Dedekind is an algebraic one. Subtraction can not be done without restriction in natural numbers $N$. If we consider the definition of multiplication of integral domain $Z, N$ with respect to subtraction is needed. It is necessary to give the definition of subtraction in $N$. Instead of starting from natural numbers, one could begin with any commutative semi-group and construct from it as the construction of the integers to obtain a commutative group. If the cancellation law does not hold in the commutative semi-group, some modifications are required. The mapping from the commutative semi-group to the commutative group is not injective and compatible with addition. In the relation between real numbers and decimals, $N$ also plays an important role.
\end{abstract}

Keywords: Well-defined, Equivalence relation, Commutative group, Cancellation law, Injective, Compatible, Archimedean property

\section{The construction and application of subtraction of natural numbers}

\subsection{Subtraction of natural numbers $N$}

Definition $\quad a=b-c \Longleftrightarrow a+c=b . \quad \forall a, b, c \in N$.

If $a=b-c$ and also $a^{\prime}=b-c$, then $a+c=b$ and $a^{\prime}+c=b$. And we have $a=a^{\prime}$ from $a+c=a^{\prime}+c$, according to the cancellation law of $N$. Hence, subtraction of $N$ is well-defined.

Besides, commutative law, association law and distribution law with respect to subtraction of $N$ are satisfied.

Commutative law: $a=b-c \Leftrightarrow a+c=b . \quad a^{\prime}=c-b \Leftrightarrow a^{\prime}+b=c$. We have $a^{\prime}+(a+c)=c \Rightarrow a^{\prime}+a=0$.

Namely, $(c-b)+(b-c)=0,(c-b)=-(b-c)$.

Association law: $a=b-c \Leftrightarrow a+c=b . \Rightarrow d+b=d+(a+c) \Rightarrow d+b=(d+a)+c \Rightarrow d+a=(d+b)-c \Rightarrow d+(b-c)=(d+b)-c$.

Distribution law: $a=b-c \Leftrightarrow a+c=b . \Rightarrow d(a+c)=d b \Rightarrow d a+d c=d b \Rightarrow d a=d b-d c \Rightarrow d(b-c)=d b-d c$.

Similarly, $a=b-c \Leftrightarrow a+c=b . \Rightarrow(a+c) d=b d \Rightarrow a d+c d=b d \Rightarrow a d=b d-c d \Rightarrow(b-c) d=b d-c d$.

According to the operations of $N$, we can prove multiplication in $Z$ is well-defined and integers form an integral domain with respect to addition and multiplication.

\subsection{The integral domain $Z$}

We should like $(a-b) \cdot(c-d)$ to be equal to $(a c+b d)-(a d+b c)$ and accordingly this leads to the following definition: $[a, b] \cdot[c, d]=[a c+b d, a d+b c]$ for $a, b, c, d \in N$

This definition is independent of the particular choice of the representative pairs.

Next we will prove $[a, b] \cdot[c, d]=[a c+b d, a d+b c]$ for $a, b, c, d \in N$ is well-defined.

If $[a, b]=\left[a^{\prime}, b^{\prime}\right],[c, d]=\left[c^{\prime}, d^{\prime}\right]$, then $[a, b]=\left[a^{\prime}, b^{\prime}\right] \Rightarrow a+b^{\prime}=a^{\prime}+b \Rightarrow a=a^{\prime}+b-b^{\prime}$.

$[c, d]=\left[c^{\prime}, d^{\prime}\right] \Rightarrow c+d^{\prime}=c^{\prime}+d \Rightarrow c=c^{\prime}+d-d^{\prime} .[a, b] \cdot[c, d]=[a c+b d, a d+b c],\left[a^{\prime}, b^{\prime}\right] \cdot\left[c^{\prime}, d^{\prime}\right]=\left[a^{\prime} c^{\prime}+b^{\prime} d^{\prime}, a^{\prime} d^{\prime}+b^{\prime} c^{\prime}\right]$.

We have $a c+b d+a^{\prime} d^{\prime}+b^{\prime} c^{\prime}=\left(a^{\prime}+b-b^{\prime}\right)\left(c^{\prime}+d-d^{\prime}\right)+b d+a^{\prime} d^{\prime}+b^{\prime} c^{\prime}=a^{\prime} c^{\prime}+a^{\prime} d-a^{\prime} d^{\prime}+b c^{\prime}+b d-b d^{\prime}-b^{\prime} c^{\prime}-$ $b^{\prime} d+b^{\prime} d^{\prime}+b d+a^{\prime} d^{\prime}+b^{\prime} c^{\prime}=a^{\prime} c^{\prime}+a^{\prime} d+b c^{\prime}+2 b d-b d^{\prime}-b^{\prime} d+b^{\prime} d^{\prime}$

$a^{\prime} c^{\prime}+b^{\prime} d^{\prime}+a d+b c=a^{\prime} c^{\prime}+b^{\prime} d^{\prime}+\left(a^{\prime}+b-b^{\prime}\right) d+b\left(c^{\prime}+d-d^{\prime}\right)=a^{\prime} c^{\prime}+b^{\prime} d^{\prime}+a^{\prime} d+b d-b^{\prime} d+b c^{\prime}+b d-b d^{\prime}=$ $a^{\prime} c^{\prime}+b^{\prime} d^{\prime}+a^{\prime} d+2 b d-b^{\prime} d+b c^{\prime}-b d^{\prime}$ 
then $(a c+b d)+\left(a^{\prime} d^{\prime}+b^{\prime} c^{\prime}\right)=\left(a^{\prime} c^{\prime}+b^{\prime} d^{\prime}\right)+(a d+b c)$.Namely, $[a c+b d, a d+b c]=\left[a^{\prime} c^{\prime}+b^{\prime} d^{\prime}, a^{\prime} d^{\prime}+b^{\prime} c^{\prime}\right]$.

That is to say, $[a, b] \cdot[c, d]=\left[a^{\prime}, b^{\prime}\right] \cdot\left[c^{\prime}, d^{\prime}\right]$.

Theorem The integers form an integral domain with respect to addition and multiplication. (that is, a commutative ring without zero divisors and with identity element).

We have proved $Z$ is a commutative group with respect to addition. Next we will consider $Z$ with respect to multiplication.

Commutative law: $[a, b] \cdot[c, d]=[a c+b d, a d+b c]=[c a+d b, c b+d a]=[c, d] \cdot[a, b] . \quad \forall[a, b],[c, d] \in Z$

Associative law:

$([a, b] \cdot[c, d]) \cdot[e, f]=[a c+b d, a d+b c] \cdot[e, f]=[(a c e+b d e)+(a d f+b c f),(a c f+b d f)+(a d e+b c e)]=[(a c e+a d f)+$ $(b c f+b d e),(a c f+a d e)+(b c e+b d f)]=[a, b] \cdot[c e+d f, c f+d e]=[a, b] \cdot([c, d] \cdot[e, f]) \quad \forall[a, b],[c, d],[e, f] \in Z$

Distribution law:

$[a, b] \cdot([c, d]+[e, f])=[a, b] \cdot[c+e, d+f]=[a(c+e)+b(d+f), a(d+f)+b(c+e)]=[a c+a e+b d+b f, a d+a f+b c+b e]$ $=[(a c+b d)+(a e+b f),(a d+b c)+(a f+b e)]=[a c+b d, a d+b c]+[a e+b f, a f+b e]=[a, b] \cdot[c, d]+[a, b] \cdot[e, f]$

Here we know $Z$ is a commutative ring.

Besides, $[1,0] \cdot[a, b]=[a, b] \cdot[1,0]=[a \cdot 1+b \cdot 0, a \cdot 0+b \cdot 1]=[a, b] . \quad \forall[a, b] \in Z$.

Next we assume there exist zero-divisors in $Z$, that is to say, $\exists[a, b] \neq[0,0]$, and $\exists[c, d] \neq[0,0]$.

$[a, b] \cdot[c, d]=[a c+b d, a d+b c]=[0,0], \quad \forall[a, b],[c, d] \in Z$.

Then $a c+b d+0=0+a d+b c, a c+b d=a d+b c, a c-a d=b c-b d, a(c-d)=b(c-d), a(c-d)-b(c-d)=$ $0,(c-d)(a-b)=0 . \Rightarrow c=d$ or $a=b$.

Which is contradictory to the assumption $[a, b] \neq[0,0],[c, d] \neq[0,0]$.

Hence, the assumption is not satisfied, there is no zero-divisors in $Z$.

Here we should also prove "If $m, n \in N$ and $m n=0$ then $m=0$ or $n=0$. $\Leftrightarrow$ If $m \neq 0$ and $n \neq 0$, then $m n \neq 0$." by induction.

Firstly, we should prove "If $m \neq 0$ and $n \neq 0$, then $m+n \neq 0$." by induction.

If $m=1,1+n=S(n) \neq 0$.

If $m=k, k+n \neq 0$.

When $m=k+1,(k+1)+n=(k+n)+1=S(k+n) \neq 0$.

So, "If $m \neq 0$ and $n \neq 0$, then $m+n \neq 0$." is proved.

If $m=1,1 \cdot n=n \neq 0$.

If $m=k, k \cdot n \neq 0$.

When $m=k+1,(k+1) \cdot n=k \cdot n+n \neq 0$.

Hence, ",'If $m, n \in N$ and $m n=0$ then $m=0$ or $n=0$." is proved.

\section{The construction of commutative group}

We begin with any commutative semi-group $H$ and construct from it as the construction of the integers to obtain a commutative group $G$. If the cancellation law does not hold in $H$, we define $(a, b) \sim(c, d)$ if and only if there is an $e$ such that $a+d+e=b+c+e$. However, in this case $\iota: H \longrightarrow G$ is not injective.

\subsection{The relation defined on $\mathrm{H} \times \mathrm{H}$}

We consider the relation $\sim$, defined on $H \times H$, by $(a, b) \sim(c, d)$ if and only if there is an $e$ such that $a+d+e=b+c+e$. We then establish that this is an equivalence relation.

It may be proved as follows:

Reflexivity: There is an $e$ such that $a+b+e=b+a+e \Rightarrow(a, b) \sim(a, b) . \quad \forall(a, b) \in H$

Symmetry: If $(a, b) \sim(c, d)$, then there is an $e$ such that $a+d+e=b+c+e$.

Hence, $c+b+e=d+a+e \Rightarrow(c, d) \sim(a, b) . \quad \forall(a, b),(c, d) \in H$

Transitivity: If $(a, b) \sim(c, d)$ and $(c, d) \sim(e, f)$ then by definition, there are $g$ and $h$ such that $a+d+g=b+c+g$ and $c+f+h=d+e+h . \quad \forall(a, b),(c, d),(e, f) \in H$

By addition we obtain $a+d+g+c+f+h=b+c+g+d+e+h$. And by letting $i=g+h+c+d$, we obtain there is an $i$ such that $a+f+i=b+e+i$, that is $(a, b) \sim(e, f)$. (We have also made use of the commutativity and associativity of addition.)

$G$ may now be defined as equivalence classes of the relation $\sim$. The class represented by $(a, b)$ is denoted by $[a, b] . G$ is a 
set of equivalence classes.

\subsection{Addition on $H \times H$}

We can define on $H \times H$ a component-wise addition, $(a, b)+(c, d):=(a+c, b+d)$.

The commutative and associative laws hold, and the zero element is $(0,0)$.

Commutative law: $(a, b)+(c, d):=(a+c, b+d)=(c+a, d+b)=(c, d)+(a, b)$.

Associative law:

$((a, b)+(c, d))+(e, f)=(a+c, b+d)+(e, f)=(a+(c+e), b+(d+f))=(a, b)+(c+e, d+f))=(a, b)+((c, d)+(e, f))$.

Zero element: $(0,0)+(a, b)=(a, b)+(0,0)=(a, b)$.

This addition is compatible with the relation $\sim$, that is to say, if $\left(a^{\prime}, b^{\prime}\right) \sim(a, b)$ and $\left(c^{\prime}, d^{\prime}\right) \sim(c, d)$ then $\left(a^{\prime}+c^{\prime}, b^{\prime}+d^{\prime}\right) \sim$ $(a+c, b+d)$.

$\left(a^{\prime}, b^{\prime}\right) \sim(a, b),\left(c^{\prime}, d^{\prime}\right) \sim(c, d) \Rightarrow a^{\prime}+b=b^{\prime}+a, c^{\prime}+d=d^{\prime}+c \Rightarrow\left(a^{\prime}+c^{\prime}\right)+(b+d)=\left(b^{\prime}+d^{\prime}\right)+(a+c)$

$\Rightarrow\left(a^{\prime}+c^{\prime}, b^{\prime}+d^{\prime}\right) \sim(a+c, b+d)$.

It is therefore meaningful to introduce in $G$, an addition $G \times G \longrightarrow G,[a, b]+[c, d]:=[a+c, b+d]$, which is likewise commutative and associative and which has $[0,0]$ as zero element.

Commutative law: $[a, b]+[c, d]:=[a+c, b+d]=[c+a, d+b]=[c, d]+[a, b]$.

Associative law:

$([a, b]+[c, d])+[e, f]=[a+c, b+d]+[e, f]=[a+(c+e), b+(d+f)]=[a+(c+e), b+(d+f)]=[a, b]+([c, d]+[e, f])$.

Zero element: $[0,0]+[a, b]=[a, b]+[0,0]=[a, b]$.

Next we will prove the addition in $G$ is well-defined.

If $[a, b]=\left[a^{\prime}, b^{\prime}\right]$ and $[c, d]=\left[c^{\prime}, d^{\prime}\right]$, we should check $[a, b]+[c, d]=\left[a^{\prime}, b^{\prime}\right]+\left[c^{\prime}, d^{\prime}\right]$.

Solution: $[a, b]=\left[a^{\prime}, b^{\prime}\right] \Rightarrow$ there is an $e$ such that $a+b^{\prime}+e=a^{\prime}+b+e$.

$[c, d]=\left[c^{\prime}, d^{\prime}\right] \Rightarrow$ there is an $f$ such that $c+d^{\prime}+f=c^{\prime}+d+f$.

Then there is a $g=e+f$ such that $a+c+b^{\prime}+d^{\prime}+g=a^{\prime}+c^{\prime}+b+d+g$.

And $[a, b]+[c, d]=[a+c, b+d],\left[a^{\prime}, b^{\prime}\right]+\left[c^{\prime}, d^{\prime}\right]=\left[a^{\prime}+c^{\prime}, b^{\prime}+d^{\prime}\right]$. Hence $[a, b]+[c, d]=\left[a^{\prime}, b^{\prime}\right]+\left[c^{\prime}, d^{\prime}\right]$.

By passing to equivalence classes we have gained more. Each $[a, b]$ has an inverse, namely $[b, a]$. We have established the following.

\subsection{Commutative group $G$}

Theorem $G$ forms a commutative group with respect to addition.

The element inverse to $\alpha \in G$ is uniquely determined, and is denoted by $-\alpha$. Subtraction in $G$ is defined by $\alpha-\beta:=\alpha+(-\beta)$.

Proof: $(1) \forall[a, b],[c, d] \in G,[a, b]+[c, d] \in G$.

(2) $\forall[a, b],[c, d] \in G,[a, b]+[c, d]=[c, d]+[a, b]$.

(3) $\forall[a, b],[c, d],[e, f] \in G,([a, b]+[c, d])+[e, f]=[a, b]+([c, d]+[e, f])$.

(4) $\forall[a, b] \in G, \exists[0,0] \in G,[0,0]+[a, b]=[a, b]+[0,0]=[a, b]$. And $[0,0]=[0,0]+[0,0]^{\prime}=[0,0]^{\prime}$, the zero element is unique.

(5) $\forall[a, b] \in G, \exists[b, a]=-[a, b] \in G,[a, b]+(-[a, b])=(-[a, b])+[a, b]=[0,0]$.

In fact, $[a, b]+[b, a]=[a+b, b+a]$ and $a+b+0=b+a+0$. Then there exists an $e=0$ such that $a+b+0+0=b+a+0+0$. Hence $[a, b]+[b, a]=[0,0]$.

Besides, $[a, b]+[c, d]=[0,0] \Rightarrow[b, a]+([a, b]+[c, d])=[b, a]+[0,0] \Rightarrow[b, a]+[a, b]+[c, d]=[b, a] \Rightarrow[c, d]=[b, a]$. The inverse of $[a, b]$ is also unique.

\subsection{The mapping from $H$ to $G$}

The mapping $\iota: H \longrightarrow G, a \longrightarrow[a, 0]$ is not injective and compatible with addition.

If the cancellation law does not hold in $H$, that is to say, there are $a, b, c \in H$ such that $a+c=b+c$ and $a \neq b$.

Then $[a, 0]=[b, 0]$ and $a \neq b$, namely, $\iota(a)=\iota(b)$ and $a \neq b$. Hence, $\iota$ is not injective.

Besides, $\iota$ is compatible with addition, because of $\iota(a)=[a, 0], \iota(b)=[b, 0], \iota(a+b)=[a+b, 0]=[a, 0]+[b, 0] \Rightarrow$ $\iota(a+b)=\iota(a)+\iota(b)$. 


\section{The relation between real numbers and decimals}

The relation between real numbers and decimals has been generally pointed out in The principle of mathematical analysis. Since the importance of application of Archimedean property of $R$ and the relationship between real numbers and decimals, the method of how to choose $n_{1}, \ldots, n_{k-1}$ of "Having chosen $n_{0}, n_{1}, \ldots, n_{k-1}$, let $n_{k}$ be the largest integer such that $n_{0}+\frac{n_{1}}{10}+\cdots+\frac{n_{k-1}}{10^{k-1}}+\frac{n_{k}}{10^{k}} \leq x$ "as been given, and the proof of "Let $E$ be the set of these numbers $n_{0}+\frac{n_{1}}{10}+\cdots+\frac{n_{k-1}}{10^{k-1}}+\frac{n_{k}}{10^{k}}(k=0,1,2, \cdots)(5)$.Then $x=$ supE."'as been indicated, which Rudin have not mentioned totally. In the proof of the two questions natural numbers also play an important role.

\subsection{The existence of $n_{0}$.}

Theorem 1.20 (a) If $x \in R, y \in R$ and $x>0$ then there is a positive integer $n$ such that $n x>y$.

Part (a) is usually referred to as the Archimedean property of $R$.

Let $x>0$ be real.

According to the Archimedean property of $R, x \in R, 1 \in R, 1>0$, then there is a positive integer $n$ such that $n \cdot 1>x$.

Hence $x \in[0,1) \cup[1,2) \cup \cdots \cup[n-1, n)$, then there is $n_{0} \in Z^{+} \cup 0$ such that $x \in\left[n_{0}, n_{0}+1\right)$.

And $n_{0}$ is the largest integer such that $n_{0} \leq x<n_{0}+1$.

3.2 The method of choosing $n_{1}, \cdots, n_{k-1}$.

$0 \leq x-n_{0}<1,0 \leq 10\left(x-n_{0}\right)<10,10\left(x-n_{0}\right) \in[0,1) \cup[1,2) \cup \cdots \cup[9,10)$.

Then there exists $n_{1} \in Z$ and $0 \leq n_{1}<10$ such that $10\left(x-n_{0}\right) \in\left[n_{1}, n_{1}+1\right)$, and $n_{1}$ is the largest integer such that $n_{1} \leq 10\left(x-n_{0}\right)<n_{1}+1, \frac{n_{1}}{10} \leq x-n_{0}<\frac{n_{1}}{10}+\frac{1}{10}, 0 \leq x-n_{0}-\frac{n_{1}}{10}<\frac{1}{10}, 0 \leq 100\left(x-n_{0}-\frac{n_{1}}{10}\right)<10$.

In the similar way, there is a largest integer $n_{2}$ such that

$0 \leq n_{2} \leq 100\left(x-n_{0}-\frac{n_{1}}{10}\right)<n_{2}+1, \frac{n_{2}}{100} \leq x-n_{0}-\frac{n_{1}}{10}<\frac{n_{2}}{100}+\frac{1}{100}, 0 \leq x-n_{0}-\frac{n_{1}}{10}-\frac{n_{2}}{100}<\frac{1}{100}, 0 \leq 1000\left(x-n_{0}-\frac{n_{1}}{10}-\frac{n_{2}}{100}\right)<10$.

There is a largest integer $n_{3}$ such that $0 \leq n_{3} \leq 1000\left(x-n_{0}-\frac{n_{1}}{10}-\frac{n_{2}}{100}\right)<n_{3}+1$.

Do the same actions till we obtain $n_{k-1}$ such that $0 \leq n_{k-1} \leq 10^{k-1}\left(x-n_{0}-\frac{n_{1}}{10}-\cdots-\frac{n_{k-2}}{10^{k-2}}\right)<n_{k-1}+1$.

3.3 The proof of $x=\operatorname{supE}$.

Let $n_{k}$ be the largest integer such that $0 \leq n_{k} \leq 10^{k}\left(x-n_{0}-\frac{n_{1}}{10}-\cdots-\frac{n_{k-1}}{10^{k-1}}\right)<n_{k}+1$.

$x-n_{0}-\frac{n_{1}}{10}-\cdots-\frac{n_{k-1}}{10^{k-1}} \geq \frac{n_{k}}{10^{k}}, x \geq n_{0}+\frac{n_{1}}{10}+\cdots+\frac{n_{k-1}}{10^{k-1}}+\frac{n_{k}}{10^{k}}$.

Let $E$ be the set of these numbers $n_{0}+\frac{n_{1}}{10}+\cdots+\frac{n_{k-1}}{10^{k-1}}+\frac{n_{k}}{10^{k}}(k=0,1,2, \cdots)(5)$

$E=\left\{n_{0}+\frac{n_{1}}{10}+\cdots+\frac{n_{k-1}}{10^{k-1}}+\frac{n_{k}}{10^{k}} \mid k=0,1,2, \cdots\right\}$

We have known $x$ is an upper bound of $E$. Next we will prove $x$ is the smallest upper bound of $E$.

$\forall y<x, x-y>0 \Rightarrow \frac{1}{x-y}>0$.

According to Archimedean property $\frac{1}{x-y} \in R, 1 \in R, 1>0$, then there is a positive integer $n$ such that $1 \cdot n>\frac{1}{x-y}$.

We let $a_{k}=n_{0}+\frac{n_{1}}{10}+\cdots+\frac{n_{k-1}}{10^{k-1}}+\frac{n_{k}}{10^{k}}, k=0,1,2, \cdots$.

$n(x-y)>1, n x-n y>1, n x-1>n y \Rightarrow y<x-\frac{1}{n}(*)$

We have known $10^{k}\left(x-\left(n_{0}+\frac{n_{1}}{10}+\cdots+\frac{n_{k-1}}{10^{k-1}}\right)\right)<n_{k+1} \Rightarrow x-\left(n_{0}+\frac{n_{1}}{10}+\cdots+\frac{n_{k-1}}{10^{k-1}}+\frac{n_{k}}{10^{k}}\right)<+\frac{1}{10^{k}} \Rightarrow x-a_{k}<\frac{1}{10^{k}}(* *)$

Next we will proof $10^{k} \geq n$ by the principle of complete induction to complete the proof. If a certain property is possessed by the number 0 (the commencement of the induction) and if, for every number $n$ which has the property, its successor also has the property(the induction step), then the property is possessed by all the natural numbers.

Step 1 When $n=0,10^{k} \geq 0$, it is satisfied.

Step 2 Assume $n=k, 10^{k} \geq k$, is satisfied.

Step 3 Then $10^{k+1}-k+1=10 \cdot 10^{k}-k-1=10^{k}-k+9 \cdot 10^{k}-1>9 \cdot 1-1=8>0.10^{k+1} \geq k+1$.

Hence, the proof of $10^{k} \geq n$ is complete.

Then $\frac{1}{10^{k}} \leq \frac{1}{n} \Rightarrow x-a_{k}<\frac{1}{n}$ (because of $\left.(* *)\right) \Rightarrow x-\frac{1}{n}<a_{k} \Rightarrow y<a_{k}$ (because of $\left.(*)\right)$.

Namely, $\forall y<x . \quad y$ is not an upper bound of $E$.

Hence, $x$ is the smallest upper bound of $E . \quad x=\sup E$.

The decimal expansion of $x$ is $n_{0} \cdot n_{1} n_{2} n_{3} \cdots(6)$.

Conversely, for any infinite decimal(6) the set of number (5)is bounded above, $\left(0 \leq n_{1}, n_{2}, \cdots, n_{k}<10\right.$ and $\left.n_{1}, n_{2}, \cdots, n_{k} \in Z\right)$ $\left(n_{0}+1\right)-a_{k}=n_{0}+1-\left(n_{0}+\frac{n_{1}}{10}+\cdots+\frac{n_{k}}{10^{k}}\right)=1-\frac{n_{1}}{10}-\frac{n_{2}}{100}-\cdots-\frac{n_{k}}{10^{k}} \geq 1-\frac{9}{10}-\frac{9}{100}-\cdots-\frac{9}{10^{k}}=1-9\left(\frac{1}{10}+\frac{1}{100}+\cdots+\frac{1}{10^{k}}\right)$ 
$=1-9 \cdot \frac{\frac{1}{10} \cdot\left(1-\frac{1}{10^{k}}\right)}{1-\frac{1}{10}}=1-\left(1-\frac{1}{10^{k}}\right)=\frac{1}{10^{k}}>0$

We have $\forall k=0,1,2, \cdots, a_{k}<n_{0}+1$.

And (6) is the decimal expansion of supE .

Acknowledgment

Acknowledgment Professor Osami Yasukura and Yutaka Saburi of Fukui University of Japan have proposed some improvements, I express here my heartfelt gratitude.

\section{References}

Akitsuki Yasuo. (1963). Abstract Algebra. Tokyo: Inc. p103-109. (in Japanese).

Heinz-Dieter Ebbinghaus, \& John H.Ewing. (1991). Numbers. (Reprint ed.). Tokyo: Springer, (Chapter 1-2).

Rudin Walter. (1976). Principles of Mathematical Analysis.(International ed.). Singapore: McGraw-Hill Book Co,(Chapter 1).

Rudin Walter. (2004). Real and Complex Analysis.(3rd ed.). China: McGraw-Hill Book Co, (Chapter 1).

Shoji Maehara. (2003). On the basis of mathematical induction. Tokyo: Inc. 2003. p79-86. (in Japanese). 